\title{
Kennedy's Disease
}

National Institute of Neurological Disorders and Stroke (NINDS)

\section{Source}

National Institute of Neurological Disorders and Stroke (NINDS). Kennedy's Disease

Information Page.

Kennedy's disease is an inherited motor neuron disease that affects males. It is one of a group of disorders called lower motor neuron disorders (which involve disruptions in the transmission of nerve cell signals in the brain to nerve cells in the brain stem and spinal cord). Onset of the disease is usually between the ages of 20 and 40, although it has been diagnosed in men from their teens to their 70s. Early symptoms include tremor of the outstretched hands, muscle cramps with exertion, and fasciculations (fleeting muscle twitches visible under the skin). Eventually, individuals develop limb weakness which usually begins in the pelvic or shoulder regions. Weakness of the facial and tongue muscles may occur later in the course of the disease and often leads to dysphagia (difficulty in swallowing), dysarthria (slurring of speech), and recurrent aspiration pneumonia. Some individuals develop gynecomastia (excessive enlargement of male breasts) and low sperm count or infertility. Still others develop non-insulin-dependent diabetes mellitus. Kennedy's disease is an x-linked recessive disease, which means the patient's mother carries the defective gene on one of her X chromosomes. Daughters of patients with Kennedy's disease are also carriers and have a 1 in 2 chance of having a son affected with the disease. Parents with concerns about their children may wish to talk to a genetic counselor. 